\section{Variación estacional de las hospitalizaciones por infarto agudo de miocardio según sexo y edad en Chile}

\author{
CAROLINA NAZZAL ${ }^{1, a, c}$, FAUSTINO ALONSO ${ }^{1, b}$
}

\section{Seasonal variation in hospital admissions due to acute myocardial infarction according to sex and age in Chile}

Background: The incidence of acute myocardial infarction (AMI) varies according to seasonality, being higher in winter. The effect of sex on this phenomenon is not clear. Aim: To evaluate the effect of seasonality in men and women hospitalized for AMI at different ages. Material and Methods: We included all patients with a primary diagnosis of AMI admitted in public and private hospitals in Chile during 2002-2011 (codes I21-I22, of the tenth international classification of diseases). We obtained data from the National Discharge databases available at the Ministry of Health website. We estimated the number of discharges per month and per seasonality (cold/template), and the Standardized Incidence Ratio (SIR) with the formula: number of observed cases/expected cases (average annual hospitalizations), stratified by sex and age ( $<50$ years, 50-64 years, 6574 years, $\geq 75$ years). We evaluated the effect of sex with binomial regressions for the different age strata. Results: We assessed 59,557 AMI hospitalizations (69\% men, with and without ST elevation segment). May, June and July (austral winter) had a SIR of 1.10; 1.12 and 1.10, respectively. Women had a 20\% excess of hospitalizations during cold seasons at any age. In men, the excess of hospitalizations increased from $9 \%$ in those aged $<50$ years to $21 \%$ in those $\geq 75$ years $(p=0.043)$. When comparing women and men, women aged $<50$ years showed the higher risk of being hospitalized during cold seasons (adjusted risk ratio $=1.06$; 95\% confidence intervals 1.01-1.13). Conclusions: Women have a stronger seasonal pattern in AMI hospitalizations than men. While this effect increases with age in men, in women it remains constant at all ages.

(Rev Med Chile 2018; 146: 1233-1240)

Key-words: Chile; Hospitalization; Myocardial Infarction; Seasons.
'Programa de Epidemiología, Institutod de Salud Poblacional. Escuela de Salud Pública, Facultad de Medicina, Universidad de

Chile. Santiago, Chile. aEnfermera matrona. ${ }^{\text {b}}$ Alumno programa Doctorado en Salud Pública, Facultad de Medicina, Universidad de Chile. Santiago, Chile.

${ }^{\mathrm{b}} \mathrm{MPH}, \mathrm{PhD}$.

Financiamiento: Trabajo financiado por el fondo de investigación del Instituto de Salud Poblacional - Escuela de Salud Pública, Facultad de Medicina, Universidad de Chile. Los autores declaran no tener conflictos de interés.

Recibido el 7 de julio de 2018, aceptado el 25 de octubre de 2018.

Correspondencia a: Carolina Nazzal Nazal Independencia 939, Independencia, Región Metropolitana, Chile. cnazzal@med.uchile.cl
E n Chile, la enfermedad coronaria es la segunda causa de muerte en ambos sexos después del accidente cerebrovascular. Se ha estudiado el efecto de la temperatura como un factor asociado a la incidencia y mortalidad por infarto agudo de miocardio (IAM) ${ }^{1-5}$. Dicha asociación ha sido bien documentada en distintos escenarios, estimándose que por cada grado Celsius que disminuye la temperatura, el riesgo de
IAM aumenta en aproximadamente $2 \%$, siendo los adultos mayores los más afectados ${ }^{4,6,7}$. Posibles mecanismos por los cuales el frío induce isquemia coronaria incluyen un efecto procoagulación y activación del sistema simpático ${ }^{3,5}$, aumento de la presión arterial y vasoconstricción ${ }^{8,9}$.

La evidencia muestra que las variaciones estacionales afectan especialmente a pacientes de edad avanzada ${ }^{2,10-12}$. Por otra parte, se ha repor- 
tado que las mujeres presentan un riesgo mayor de incidencia y muerte por IAM en climas fríos ${ }^{13}$, lo que se ha explicado porque éstas sufren de infartos en forma más tardía que los hombres ${ }^{14}$. También se ha identificado mayor susceptibilidad a las variaciones estacionales en pacientes de bajo nivel socioeconómico, asociado a las condiciones de urbanización, vivienda y calefacción ${ }^{15,16}$.

El estudio de la estacionalidad cobra hoy nuevamente vigencia, debido a los posibles efectos del cambio climático en la salud de la población. Chile ha experimentado cambios en la temperatura en la última década, registrándose un aumento de la temperatura mínima en la zona Centro-Norte (con un máximo de $0,47^{\circ} \mathrm{C} /$ década), en cambio una disminución en la zona Sur (máximo registrado $-0,14 \mathrm{C} /$ década $)^{17}$. Al respecto, la comunidad científica recalca la importancia de estudiar cómo estos cambios afectarían a distintos grupos poblacionales, resaltando la importancia de abordar posibles inequidades ${ }^{18,19}$.

El objetivo de este estudio es evaluar el efecto de la variación estacional en la incidencia de hospitalizaciones por IAM, según sexo y edad. Para ello, se analizará esta relación en distintos grupos etarios, incorporando el nivel socioeconómico (medido a través del seguro de salud) como variable de ajuste en el análisis.

\section{Material y Métodos}

\section{Población y diseño}

Estudio de base poblacional en mayores de 15 años hospitalizados por un primer IAM entre 1 enero de 2002 y 31 de diciembre de 2011 en Chile. Los casos de IAM se identificaron de las bases de egresos hospitalarios nacionales de los años 2002 al 2011 que registran los egresos de todos los hospitales públicos y privados a nivel nacional. Cabe señalar que el 2002 se implementó un sistema de validación de diagnósticos y datos, ejecutado en el Departamento de Estadísticas e Información en Salud del Ministerio de Salud chileno (DEIS), mejorando su calidad y completitud. Los casos de IAM se seleccionaron utilizando el código I 21 de la Décima versión de la Clasificación Internacional de Enfermedades (CIE-10). En caso de ocurrencia de más de un evento en un mismo paciente durante el período de estudio (IAM recurrente), se seleccionó sólo el primer evento.
Durante el período analizado se identificaron 72.108 hospitalizaciones por IAM en 60.651 pacientes; $28,3 \%$ fueron IAM recurrentes. Se excluyeron 291 hospitalizaciones por falta de la fecha de egreso, 5 por falta de fecha de ingreso, 6 sin información de edad, 38 por registrar una estadía hospitalaria mayor a 365 días, 5 ocurridos en menores de 15 años y 1.101 debido a falta de información sobre seguro de salud, que para este análisis se empleó como indicador de nivel socioeconómico. 51.350 pacientes presentaron un solo IAM y 9.301 pacientes más de uno (correspondiendo a 20.413 hospitalizaciones, de las cuales se excluyeron 11.112 manteniendo sólo el primer ingreso para el análisis).

\section{Proceso de validación de datos y variables}

Se validó la información entregada respecto a la estructura y patrón de los datos; se identificaron los valores perdidos y aberrantes, así como la distribución de duplicados respecto al identificador único para cada ciudadano chileno (RUT). Las variables que se validaron y codificaron fueron: seguro de salud (público o privado), comuna, región, mes de atención, fecha de egreso, días de estadía, diagnóstico de egreso, edad, sexo y condición de egreso (vivo o muerto). Respecto a la información faltante para la variable sexo, en el caso de pacientes con uno o dos egresos se consultó directamente al DEIS. En aquellos pacientes con más de dos egresos se procedió a buscar la información en la misma base de datos. Para seguro de salud se imputó o mantuvo la información respecto al primer egreso. Finalmente, se eliminaron de la base aquellos pacientes que no tenían información de sexo, seguro de salud, diagnóstico y fecha de egreso y se evaluó que esta pérdida de información no afectara el patrón de los datos.

Para estratificar el nivel socioeconómico se agruparon los pacientes según el tipo de seguro de salud: privado (Instituciones de Salud Previsional, ISAPRE $\mathrm{u}$ otros seguros privados y de Fuerzas Armadas) o público (Fondo Nacional de Salud, FONASA). El seguro público clasifica a los beneficiarios en cuatro tramos de acuerdo su ingreso mensual -definido como la suma de todos los ingresos formales que recibe cada mes-. A los tramos más bajos, FONASA A y B, pertenecen los afiliados que no tienen ningún ingreso o éste no supera el ingreso mínimo mensual; y a los tramos superiores, $\mathrm{C}$ y $\mathrm{D}$, aquellos que exceden el ingreso 
mínimo mensual. Los pacientes FONASA A y B reciben atención de salud gratuita en hospitales del sector público, en cambio los grupos FONASA $\mathrm{C}$ y D deben realizar un copago. Los pacientes de grupos B, C o D pueden optar a prestaciones en algunas entidades privadas mediante un copago. De acuerdo con la Encuesta Nacional de Caracterización Socioeconómica (CASEN) de 2012, 80,1\% de la población chilena pertenece al seguro público, $13,2 \%$ a privados (ISAPRES), $3,8 \%$ a otros seguros privados y en $3,0 \%$ se desconoce o no están afilados a ningún seguro ${ }^{20}$. Para este análisis se agruparon los pacientes en: seguro privado (nivel socioeconómico alto), seguro público FONASA C y D (nivel socioeconómico medio) y FONASA A y B (nivel socioeconómico bajo).

\section{Análisis estadístico}

Para corregir el efecto de la diferencia en el número de días de cada mes se estandarizó el número de casos mensuales con la fórmula: número casos/ días del mes x 30. Se describió el número de casos mensuales estandarizados por sexo, categorizados en cuatro grupos etarios: 15 a 49 años, 50 a 64 años, 65 a 74 años y 75 o más años. La variación estacional se analizó por mes y por estación. Se consideró estación fría al período entre los meses de mayo y agosto; y estación calurosa a la comprendida entre los meses de diciembre a marzo (de acuerdo al registro de temperatura promedio mensual de la Dirección Meteorológica de Chile de los años del estudio) ${ }^{21}$. Si bien Chile presenta diversos climas y algunas ciudades son costeras, en la mayoría es posible observar estaciones frías y calurosas. Para cada grupo etario, y en forma separada para hombres y mujeres, se estimó una razón estandarizada de incidencia mensual de hospitalizaciones (REI mensual) y por estación (REI estación) calculada con la fórmula: número de casos observados/ número de casos esperados. El promedio anual de hospitalizaciones por IAM se utilizó como número de casos esperados.

\section{Estimación del efecto de la variación estacional en la incidencia de hospitalizaciones según edad $y$ sexo}

Se calculó el cociente entre la REI de estación fría y la REI de estación calurosa (CFT: cociente frío/caluroso) en cada grupo de edad para hombres y mujeres por separado. Para evaluar el tamaño del efecto de la variación estacional se calculó la diferencia absoluta de los cocientes frío/caluroso entre mujeres y hombres: $\Delta=$ (CFT mujeres - CFT hombres $)^{\star} 100$. El resultado expresa el porcentaje de hospitalizaciones en exceso que se registró en la estación fría. Con test de tendencia para variables categóricas desarrollado por Cuzick ${ }^{22}$ se testeó la hipótesis nula de igualdad de los cocientes entre las cuatro categorías de edad en cada sexo.

\section{Análisis multivariado}

Para evaluar el efecto del sexo en la asociación entre la variación estacional y la incidencia de hospitalizaciones por IAM se calcularon modelos lineales generalizados con modelo de distribución binomial múltiple ajustados por nivel socioeconómico. Se estimaron los riesgos relativos (RR) y sus intervalos de confianza de 95\% (IC95\%). Se consideró un nivel de significación de $5 \%$ a dos colas y se empleó el software Stata $13,1^{23}$. El proyecto fue aprobado por el Comité de Ética de la Facultad de Medicina de la Universidad de Chile.

\section{Resultados}

Se analizaron 59.557 pacientes con hospitalizaciones fatales y no fatales por IAM con y sin elevación del segmento ST (41.009 hombres y 18.548 mujeres). La mediana de edad fue 64 años (percentil 25: 54 años, percentil 75: 74 años); los hombres presentaron una mediana de 61 años (5474 años) y las mujeres de 70 años (59-79 años). En hombres, el mayor porcentaje de hospitalizaciones ocurrió entre 50 y 64 años, mientras que en mujeres en el grupo de 75 y más años (Tabla 1). En las mujeres, $71,5 \%$ pertenecía a FONASA A y B en comparación a $59 \%$ en los hombres. El promedio anual de hospitalizaciones fue 1.855 en mujeres y 4.101 en hombres. Las REI mayores a 1 se observaron entre mayo y agosto en las mujeres y entre mayo y septiembre en los hombres (Figura 1).

\section{Análisis estratificado por edad y sexo}

En la Figura 2 se muestran las REI mensuales para cada grupo de edad estratificadas por sexo. En el grupo de $<50$ años las mujeres presentan REI mayores que los hombres en los meses fríos, diferencia que disminuyó en la medida que avanza la edad. Los cocientes frío/caluroso para los grupos de edad y sexo se describen en la Tabla 2. El análisis de tendencia mostró que el efecto de la variación 
Tabla 1. Características socio-demográficas de los pacientes hospitalizados por IAM según sexo, Chile $2002-2011$

\begin{tabular}{|c|c|c|c|}
\hline & $\begin{array}{c}\text { Total }(n=59.557) \\
n(\%)\end{array}$ & $\begin{array}{c}\text { Hombres }(n=41.009) \\
n(\%)\end{array}$ & $\begin{array}{c}\text { Mujeres }(n=18.548) \\
n(\%)\end{array}$ \\
\hline \multicolumn{4}{|l|}{ Edad } \\
\hline$<50$ años & $9.324(15,6)$ & $7.560(18,4)$ & $1.764(9,5)$ \\
\hline 50-64 años & $21.495(36,1)$ & $16.540(40,3)$ & $4.955(26,7)$ \\
\hline 65-74 años & $14.578(24,5)$ & $9.603(23,4)$ & $4.975(26,8)$ \\
\hline$\geq 75$ años & $14.160(23,8)$ & $7.306(17,8)$ & $6.854(40,0)$ \\
\hline \multicolumn{4}{|l|}{ Seguro salud } \\
\hline Fonasa A/B & $37.443(62,9)$ & $24.179(59,0)$ & $13.264(71,5)$ \\
\hline Fonasa C/D & $11.427(19,2)$ & $8.785(21,4)$ & $2.642(14,2)$ \\
\hline Isapre y otros & $10.687(17,9)$ & $8.045(19,6)$ & $2.642(14,2)$ \\
\hline
\end{tabular}

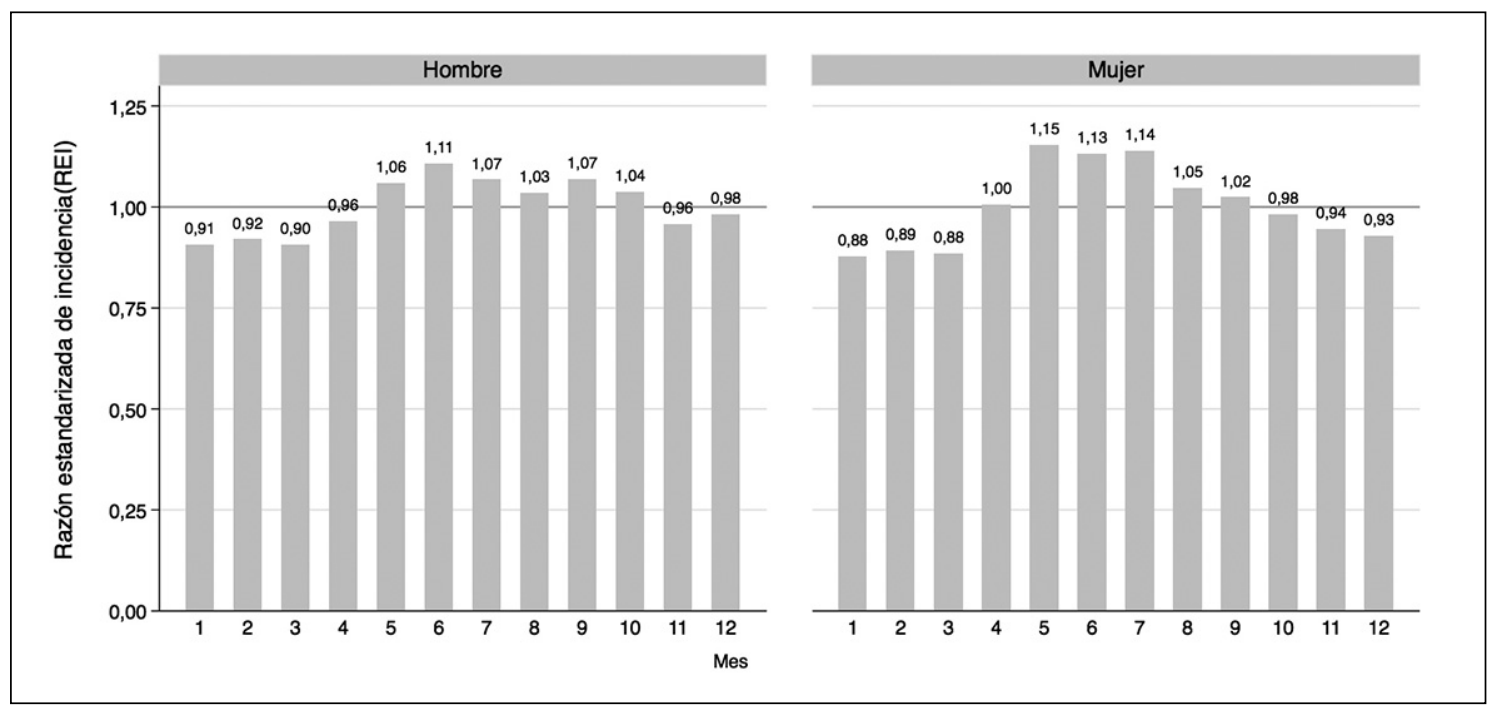

Figura 1. Razón estandarizada de incidencia por infarto agudo al miocardio según mes de ocurrencia y sexo en Chile.

Tabla 2. Efecto de la variación estacional en la incidencia de hospitalizaciones según edad y sexo: Chile 2002-2011

\begin{tabular}{|c|c|c|c|}
\hline \multirow[t]{2}{*}{ Grupo etario } & \multicolumn{2}{|c|}{ Cociente frío/caluroso (CFC) } & \multirow{2}{*}{$\begin{array}{c}\text { Diferencia absoluta } \\
\text { del CFC }\end{array}$} \\
\hline & $\begin{array}{c}\text { Hombres } \\
n=41.009\end{array}$ & $\begin{array}{c}\text { Mujeres } \\
n=18.548\end{array}$ & \\
\hline$<50$ años & 0,09 & 0,23 & $14 \%$ \\
\hline 50 - 64 años & 0,13 & 0,20 & $7 \%$ \\
\hline 65 - 74 años & 0,12 & 0,21 & $9 \%$ \\
\hline 75 y más años & 0,21 & 0,25 & $4 \%$ \\
\hline
\end{tabular}

$p$ tendencia para los distintos grupos etarios: 0,043 en hombres y 0,763 en mujeres. Cociente frío caluroso (CFC) $=$ REI invierno/ REI verano. Diferencia absoluta del CFC $(\triangle)=$ CFC mujeres - CFC hombres. 

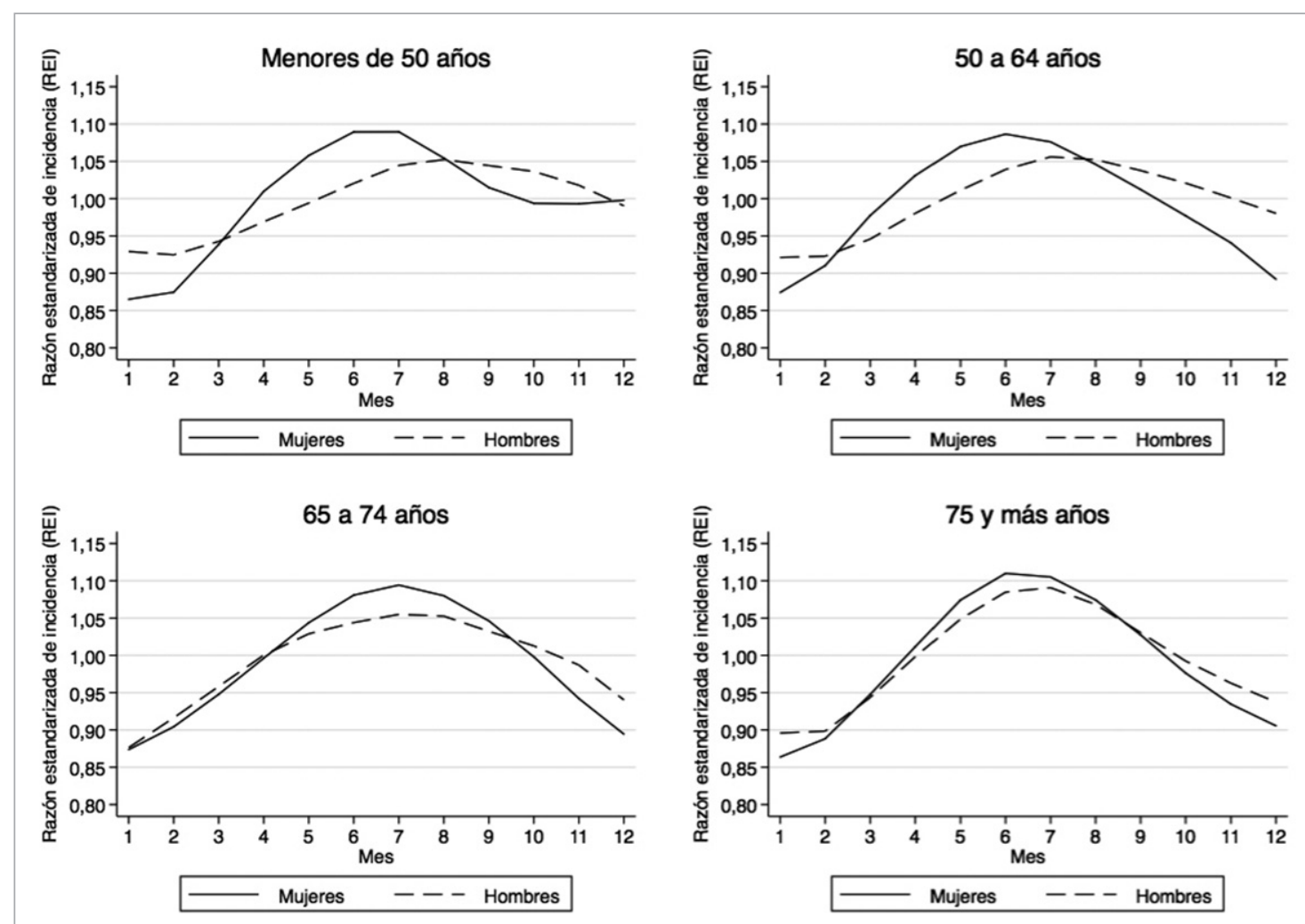

Figura 2. Razón estandarizada de incidencia por infarto agudo al miocardio según mes de ocurrencia para hombres y mujeres de acuerdo al grupo etario en Chile. ¿Qué se sabe del tema? La variación estacional tiene un efecto en la incidencia de hospitalizaciones por IAM, siendo mayor en los meses fríos, la que aumenta con la edad. ¿Qué aporta de nuevo? El efecto estacional sobre la incidencia de hospitalizaciones por IAM en los hombres aumenta con la edad. En las mujeres el efecto de la variación estacional es mayor en comparación a los hombres y se manifiesta en todas las edades.

estacional aumentó significativamente con la edad en los hombres (CFT $=0,09$ en $<50$ años a 0,21 en $\geq 75$ años; $p$ de tendencia $=0,043, Z=2,02$ ), en cambio, las mujeres presentaron consistentemente mayor incidencia de hospitalizaciones en la estación fría, independiente de la edad ( $\mathrm{p}$ de tendencia $=0,673, Z=0,42)$. La diferencia en el número de hospitalizaciones en estación fría entre mujeres y hombres fue $14 \%$ en el grupo $<50$ años, diferencia que se redujo a $4 \%$ en los $\geq 75$ años.

\section{Análisis multivariado}

En el análisis ajustado las mujeres $<50$ años presentaron $6 \%$ más riesgo de incidencia de hospitalizaciones por IAM en la estación fría, comparadas con los hombres de la misma edad $(\mathrm{RR}=1,06$; IC95\%: 1,01-1,13). La diferencia de riesgo entre mujeres y hombres se redujo según avanzó la edad: 3\% para las mujeres de 50-64 años $(\mathrm{p}=0,088), 4 \%$ en las de 65-74 años $(\mathrm{p}=0,057)$, y $1 \%$ en $\geq 75$ años $(\mathrm{p}=0,416)$ (Tabla 3$)$.

\section{Discusión}

En este estudio, que incluye una gran muestra poblacional con representatividad nacional, se verifica la presencia de la variación estacional en la incidencia de hospitalizaciones por IAM, con un alza en los meses fríos correspondiente a la estación de otoño-invierno. El principal aporte al conocimiento es describir que este exceso de hospitalizaciones por IAM en otoño-invierno aumenta con la edad en los hombres, mientras que en las mujeres es constantemente alto, independiente de la edad. 
Tabla 3. Riesgos relativos (IC 95\%) de hospitalizaciones por IAM en estación fría en mujeres comparadas con hombres según edad, Chile 2002-2011

\begin{tabular}{|lcccc|}
\hline & RR crudo (IC 95\%) & P & *RR ajustado (IC 95\%) & P \\
\hline$<50$ años & $1,07(1,01-1,12)$ & 0,029 & $1,06(1,01-1,13)$ & 0,032 \\
\hline $50-64$ años & $1,03(1,00-1,07)$ & 0,085 & $1,03(0,99-1,06)$ & 0,088 \\
\hline $65-74$ años & $1,04(1,00-1,08)$ & 0,034 & $1,04(1,00-1,08)$ & 0,057 \\
\hline 75 y más años & $1,01(0,98-1,05)$ & 0,373 & $1,01(0,97-1,05)$ & 0,416 \\
\hline
\end{tabular}

*Ajustado por seguro de salud.

En estudios previos se ha reportado la asociación del frío como factor de riesgo para IAM en mujeres, especialmente en climas calurosos, explicada principalmente por la mayor edad de presentación del IAM en las mujeres ${ }^{13,14}$. Al estratificar por edad, nuestro estudio aporta evidencia identificando a las mujeres jóvenes como más susceptibles a la variación estacional, con un riesgo significativamente mayor de hospitalizaciones en estación fría, en comparación a los hombres de su misma edad. Este hecho podría explicarse porque la baja temperatura afecta particularmente la disfunción endotelial ${ }^{24-27}$, la cual se asocia mayormente a enfermedad coronaria en mujeres. Ellas presentan más frecuentemente enfermedad microvascular no obstructiva ${ }^{28-30}$. Nuestros resultados difieren de lo reportado por otros autores, quienes muestran efectos similares en mujeres y hombres ${ }^{31,32}$; sin embargo, estos trabajos no analizan en forma separada el efecto del sexo en diferentes grupos etarios.

En relación a la edad como factor de riesgo, nuestros resultados son concordantes con reportes previos, encontrándose que los pacientes de mayor edad se afectan mayormente por la variación estacional, lo que podría estar asociado a la incidencia de infecciones respiratorias, susceptibilidad a la contaminación ambiental y a su menor capacidad de termorregulación ${ }^{2,7,10,33,34}$. Esta mayor susceptibilidad en pacientes de 75 o más años afecta a ambos sexos, lo que se traduce en una diferencia de hospitalizaciones en la estación fría entre mujeres y hombres sólo de 4\%.

Como limitaciones del estudio se consideran: las bases de datos de egresos hospitalarios tienen primariamente un fin administrativo y, por lo tanto, no están exentas de errores de codificación de diagnósticos; no podemos afirmar que todos los pacientes sean efectivamente un primer episodio de IAM para aquellos casos con hospitalizaciones ocurridas previamente al período incluido en este análisis; no se pudo identificar los IAM con y sin elevación del segmento ST, ya que dicha información no está en la base de datos empleada. Hay autores que han reportado el efecto de la variación de clima frío sólo en los IAM con elevación del segmento $\mathrm{ST}^{35}$. En nuestro estudio, al no poder diferenciar estos tipos de infartos, el efecto de la estacionalidad estaría subestimado al incluir ambas entidades en el análisis. En cuarto lugar, no se disponía de datos adecuados de contaminación ambiental por material particulado para todo el período, la cual se asocia con aumento del riesgo de enfermedades cardiovasculares en ambos sexos, especialmente, en pacientes añosos ${ }^{36-40}$. Se han reportado fracciones atribuibles entre $0,6 \%$ y $4,5 \%$, dependiendo del contaminante ${ }^{41}$. Al respecto, las características de la cuenca de Santiago, donde reside $40 \%$ de la población chilena, así como la calefacción por leña en las grandes ciudades de la zona sur, hacen que durante los meses fríos aumente la contaminación por material particulado en las grandes ciudades ${ }^{42,43}$. Sin embargo, hemos evaluado el efecto de la variación estacional, sin establecer que la relación estudiada sea consecuencia sólo de los cambios de temperatura, por lo que no afectaría los estimadores calculados. Otra limitación es que los resultados sólo aplican a pacientes hospitalizados, pero hemos decidido no emplear los casos que fallecen fuera del hospital en consideración a la validez del diagnóstico ${ }^{44}$. Por último, tampoco se dispuso de información sobre comorbilidades que pudiesen haber sido incorporadas como variables de ajuste.

Estos resultados entregan evidencia que permite avanzar en el conocimiento de las diferencias de 
la enfermedad coronaria en mujeres y hombres. El reconocimiento de subgrupos de mayor riesgo frente a los cambios estacionales permite implementar estrategias individuales en estos pacientes. Estas podrían incluir el uso de ropa adecuada en días fríos, favorecer el uso de fármacos vasodilatadores en población joven, o reducir la exposición a cambios bruscos de temperatura al transitar entre espacios cerrados y abiertos ${ }^{6}$.

\section{Conclusiones}

La variación estacional tiene un efecto en la incidencia de hospitalizaciones por IAM, siendo mayor en los meses fríos. El riesgo aumenta con la edad en los hombres, sin embargo, en las mujeres el efecto estacional se presenta desde edades tempranas y se mantiene a lo largo de la vida. La variación estacional constituye un factor de riesgo modificable que debe ser considerado en prevención cardiovascular, especialmente para los grupos de mayor riesgo.

\section{Referencias}

1. Anderson TW, Le Riche WH. Cold weather and myocardial infarction. Lancet 1970; 1 (7641): 291-6.

2. Mercer JB. Cold-an underrated risk factor for health. Environ Res 2003; 92 (1): 8-13.

3. Claeys MJ, Rajagopalan S, Nawrot TS, Brook RD. Climate and environmental triggers of acute myocardial infarction. Eur Heart J 2016.

4. Bhaskaran K, Hajat S, Haines A, Herrett E, Wilkinson $\mathrm{P}$, Smeeth L. Short term effects of temperature on risk of myocardial infarction in England and Wales: time series regression analysis of the Myocardial Ischaemia National Audit Project (MINAP) registry. BMJ 2010; 341: c3823.

5. Bhaskaran K, Hajat S, Haines A, Herrett E, Wilkinson $\mathrm{P}$, Smeeth L. Effects of ambient temperature on the incidence of myocardial infarction. Heart 2009; 95 (21): 1760-9.

6. Liu C, Yavar Z, Sun Q. Cardiovascular response to thermoregulatory challenges. Am J Physiol Heart Circ Physiol 2015; 309 (11): H1793-812.

7. Vasconcelos J, Freire E, Almendra R, Silva GL, Santana P. The impact of winter cold weather on acute myocardial infarctions in Portugal. Environ Pollut 2013; 183: 14-8.
8. Cheng X, Su H. Effects of climatic temperature stress on cardiovascular diseases. Eur J Intern Med. 2010; 21 (3): 164-7.

9. Fares A. Winter cardiovascular diseases phenomenon. N Am J Med Sci 2013; 5 (4): 266-79.

10. Sheth T, Nair C, Muller J, Yusuf S. Increased winter mortality from acute myocardial infarction and stroke: the effect of age. J Am Coll Cardiol 1999; 33 (7): 1916-9.

11. González Hernández E, Cabades O’Callaghan A, Cebrian Domenech J, López Merino V, Sanjuan Manez R, Echanove Errazti I, et al. [Seasonal variations in admissions for acute myocardial infarction. The PRIMVAC study]. Rev Esp Cardiol 2004; 57 (1): 12-9.

12. Hajat S, Kovats RS, Lachowycz K. Heat-related and cold-related deaths in England and Wales: who is at risk? Occup Environ Med 2007; 64 (2):93-100.

13. Barnett AG, Dobson AJ, McElduff P, Salomaa V, Kuulasmaa K, Sans S. Cold periods and coronary events: an analysis of populations worldwide. J Epidemiol Community Health 2005; 59 (7): 551-7.

14. Grech V, Aquilina O, Pace J. Gender differences in seasonality of acute myocardial infarction admissions and mortality in a population-based study. J Epidemiol Community Health 2001; 55 (2): 147-8.

15. Kwon BY, Lee E, Lee S, Heo S, Jo K, Kim J, et al. Vulnerabilities to Temperature Effects on Acute Myocardial Infarction Hospital Admissions in South Korea. Int J Environ Res Public Health 2015; 12 (11): 14571-88.

16. O’Neill MS, Zanobetti A, Schwartz J. Modifiers of the temperature and mortality association in seven US cities. Am J Epidemiol 2003; 157 (12): 1074-82.

17. Dirección general de aeronáutica civil, Dirección meteorológica de Chile, Climatología-oficina de cambio climático. http://archivos.meteochile.gob.cl/portaldmc/ meteochile/documentos/ResumenClimaticoChile2016. pdf 2016 [citado el 8 mayo de 2017]. Disponible en: http://archivos.meteochile.gob.cl/portaldmc/meteochile/ documentos/ResumenClimaticoChile2016.pdf.

18. De Blois J, Kjellstrom T, Agewall S, Ezekowitz JA, Armstrong PW, Atar D. The Effects of Climate Change on Cardiac Health. Cardiology 2015; 131 (4): 209-17.

19. Watts N, Adger WN, Agnolucci P, Blackstock J, Byass P, Cai W, et al. Health and climate change: policy responses to protect public health. Lancet 2015; 386 (10006): 1861-914.

20. Ministerio de Desarrollo Social-Gobierno de Chile. Encuesta de Caracterización Socioeconómica Nacional, CASEN 2011. 2012.

21. Dirección General de Aeronáutica Civil, Chile DMd.

22. Cuzick J. A Wilcoxon-type test for trend. Stat Med 1985; 4 (1): 87-90. 
23. StataCorp. Stata Statistical Software College Station, TX. Release 12. 1 ed: StataCorp LP; 2011.

24. Zhu Z, Zhu S, Zhu J, van der Giet M, Tepel M. Endothelial dysfunction in cold-induced hypertensive rats. Am J Hypertens 2002; 15 (2 Pt 1): 176-80.

25. Widlansky ME, Vita JA, Keyes MJ, Larson MG, Hamburg NM, Levy D, et al. Relation of season and temperature to endothelium-dependent flow-mediated vasodilation in subjects without clinical evidence of cardiovascular disease (from the Framingham Heart Study). Am J Cardiol 2007; 100 (3): 518-23.

26. Nawrot TS, Staessen JA, Fagard RH, Van Bortel LM, Struijker-Boudier HA. Endothelial function and outdoor temperature. Eur J Epidemiol 2005; 20 (5): 407-10.

27. Ebner A, Poitz DM, Alexiou K, Deussen A. Secretion of adiponectin from mouse aorta and its role in cold storage-induced vascular dysfunction. Basic Res Cardiol 2013; 108 (6): 390.

28. Kuruvilla S, Kramer CM. Coronary microvascular dysfunction in women: an overview of diagnostic strategies. Expert Rev Cardiovasc Ther 2013; 11 (11): 1515-25.

29. Kothawade K, Bairey Merz CN. Microvascular coronary dysfunction in women: pathophysiology, diagnosis, and management. Curr Probl Cardiol 2011; 36 (8): 291-318.

30. Bairey Merz CN, Shaw LJ, Reis SE, Bittner V, Kelsey $\mathrm{SF}$, Olson $\mathrm{M}$, et al. Insights from the NHLBI-Sponsored Women's Ischemia Syndrome Evaluation (WISE) Study: Part II: gender differences in presentation, diagnosis, and outcome with regard to gender-based pathophysiology of atherosclerosis and macrovascular and microvascular coronary disease. J Am Coll Cardiol 2006; 47 (3 Suppl): S21-9.

31. Fischer T, Lundbye-Christensen S, Johnsen SP, Schonheyder HC, Sorensen HT. Secular trends and seasonality in first-time hospitalization for acute myocardial infarction-a Danish population-based study. Int J Cardiol 2004; 97 (3): 425-31.

32. Abrignani MG, Corrao S, Biondo GB, Renda N, Braschi A, Novo G, et al. Influence of climatic variables on acute myocardial infarction hospital admissions. Int J Cardiol 2009; 137 (2): 123-9.

33. Swampillai J, Wijesinghe N, Sebastian C, Devlin GP. Seasonal Variations in Hospital Admissions for ST-Elevation Myocardial Infarction in New Zealand. Cardiol Res 2012; 3 (5): 205-8.

34. Zheng S, Wang M, Li B, Wang S, He S, Yin L, et al. Gender, Age and Season as Modifiers of the Effects of Diurnal Temperature Range on Emergency Room Admissions for Cause-Specific Cardiovascular Disease among the Elderly in Beijing. Int J Environ Res Public Health 2016; 13 (5).

35. Leibowitz D, Planer D, Weiss T, Rott D. Seasonal variation in myocardial infarction is limited to patients with ST-elevations on admission. Chronobiol Int 2007; 24 (6): 1241-7.

36. Wang C, Tu Y, Yu Z, Lu R. PM2.5 and Cardiovascular Diseases in the Elderly: An Overview. Int J Environ Res Public Health 2015; 12 (7): 8187-97.

37. Pope CA, 3rd, Burnett RT, Thurston GD, Thun MJ, Calle EE, Krewski D, et al. Cardiovascular mortality and long-term exposure to particulate air pollution: epidemiological evidence of general pathophysiological pathways of disease. Circulation 2004; 109 (1): 71-7.

38. Brook RD, Rajagopalan S, Pope CA, 3rd, Brook JR, Bhatnagar A, Diez-Roux AV, et al. Particulate matter air pollution and cardiovascular disease: An update to the scientific statement from the American Heart Association. Circulation 2010; 121 (21): 2331-78.

39. Newby DE, Mannucci PM, Tell GS, Baccarelli AA, Brook $\mathrm{RD}$, Donaldson $\mathrm{K}$, et al. Expert position paper on air pollution and cardiovascular disease. Eur Heart J 2015; 36 (2): 83-93b.

40. Miller KA, Siscovick DS, Sheppard L, Shepherd K, Sullivan JH, Anderson GL, et al. Long-term exposure to air pollution and incidence of cardiovascular events in women. N Engl J Med 2007; 356 (5): 447-58.

41. Mustafic H, Jabre P, Caussin C, Murad MH, Escolano $S$, Tafflet $M$, et al. Main air pollutants and myocardial infarction: a systematic review and meta-analysis. JAMA 2012; 307 (7): 713-21.

42. Morales RGE. Contaminación atmosférica urbana: episodios críticos de contaminación ambiental en la ciudad de Santiago 2006 [citado el 11 mayo de 2017]. Disponible en: https://books.google.cl/books?id=HdeX6SWHBW8C\&dq=temperatura + fria $++y+$ contaminacion+ambiental+en+chile\&source=gbs_navlinks_s.

43. Sanhueza P, Vargas C, Mellado P. [Impact of air pollution by fine particulate matter (PM10) on daily mortality in Temuco, Chile]. Rev Med Chile 2006; 134 (6): 754-61.

44. McCormick N, Lacaille D, Bhole V, Avina-Zubieta JA. Validity of myocardial infarction diagnoses in administrative databases: a systematic review. PLoS One 2014; 9 (3): e92286. 